\title{
Article
}

\section{Peristaltic Tube Flow of a Giesekus Fluid}

\author{
Aamar Kamal ABBASI ${ }^{*}, \dagger$, Nasir ALI ${ }^{* *}$, Muhammad SAJID ${ }^{* * *}$, \\ Iftikhar AHMAD", and Sadaqut HUSSAIN ${ }^{* *}$ \\ "Department of Mathematics, University of Azad Jammu \& Kashmir, \\ Muzaffarabad 13100, Pakistan \\ ${ }^{* *}$ Department of Mathematics and Statistics, International Islamic University \\ Islamabad 44000, Pakistan \\ ${ }^{* * *}$ Theoretical Physics Division, PINSTECH, P.O. Nilore, \\ Islamabad 44000, Pakistan \\ (Received : October 20, 2015)
}

\begin{abstract}
The objective of this work is to study the peristaltic motion of an incompressible Giesekus fluid in a circular cylindrical tube. The problem is modeled in a fixed frame of reference and then transformed into a frame that moves with the wave speed. The most widely taken assumptions of long wavelength and low Reynolds number are applied in the wave frame. Both exact and approximate solutions of governing equation for stream function are obtained at each cross-section by solving nonlinear algebraic equations. The comparison of the two solutions is presented graphically. The exact solution is then used to analyze the effects of parameters of interest on velocity profile, pressure rise per wavelength and trapping phenomenon. The results disclose that the magnitude of velocity increases in the middle-most region of the tube whereas it decreases in the vicinity of wall with increasing the Giesekus model parameters. It is also observed that the size and circulation of the trapped bolus decrease with increasing the Giesekus model parameters. Moreover, much greater mixing is realized in a plane channel than in a circular tube.
\end{abstract}

Key Words: Giesekus fluid / Peristaltic transport / Long wavelength / Circular tube

\section{INTRODUCTION}

Peristalsis is the characteristic of digestive tract and many other tubular structures which helps in the movement of their contents. Peristalsis is involved in many biological fluids transport such as, transport of urine from kidney to bladder through ureter, vassomotion of small blood vessels such as arterioles, venules and capillaries, movement of bile from the gall-bladder into the duodenum, movement of chyme in intestine, mixing of food in stomach and in many other biological systems. Peristalsis has been exploited for engineering purposes like heart-lung machine that is used in open heart surgery to support the body during the surgical procedure while the heart is stopped. The heart-lung machine is often referred to as the "pump" and it does the work of the heart and lungs during the operation.

The experimental investigation of Latham ${ }^{1)}$ provided a base to study the peristaltic flow. Several theoretical and experimental investigations have been made afterward

$\leftarrow$ Corresponding author.

E-mail: aamir_mathematics@yahoo.com to understand peristaltic action in different geometries by incorporating the effects of heat and mass transfer, magnetohydrodynamic, slip condition etc. The earliest models due to Fung and Yih $^{2)}$ and Shapiro et al. ${ }^{3)}$ describe the peristaltic transport in a two-dimensional channel/tube. These models explained the biologically and medically important phenomenon of reflux. The function of healthy ureter was analyzed by Lykoudis and Roos ${ }^{4)}$ in terms of a fluidmechanical peristaltic model under the approximations of large wavelength and low Reynolds number. Their basic equations were reduced to those obtained by using theory of lubrication. The assumption of low Reynolds number was also followed by Pozrikidis ${ }^{5)}$ to study the peristaltic flow of Newtonian fluid in a channel using boundary integral method for analysis. A numerical investigation of peristaltic flow without using long wavelength and low Reynolds number assumptions was carried out by Takabatake et al.. ${ }^{67)}$ They discussed the reflux and trapping phenomena for large Reynolds number in both channel and tube geometry. In above mentioned studies ${ }^{1-7)}$, the focus was to discuss the peristaltic flow by treating the fluid as Newtonian. Since most of the physiological fluids, for instance, blood, chyme, cervical mucus, synovial oil etc. are 
non-Newtonian, therefore peristaltic flow of non-Newtonian fluids have also received interests of researchers. In the pioneering works on peristaltic transport of non-Newtonian fluids ${ }^{8-11)}$ only limited information was available because the additional nonlinear terms appearing in the equations of motion rendered the problem more difficult to solve. Because of non-availability of universal model for description of all types of non-Newtonian behavior, many fluid models have been proposed in the literature. Such models mainly predict non-Newtonian effects like viscoelasticity, plasticity, shearthinning and thickening etc. The effects of shear-thinning/ thickening in context of peristalsis were discussed by Siddiqui and Schwarz ${ }^{12)}$, Hayat et al. ${ }^{13)}$ and Haroun. ${ }^{14)}$ In these investigations, Siddiqui and Schwarz ${ }^{12)}$ analyzed the peristaltic flow problem in a symmetric channel for a third order fluid and concluded that pumping rate of shear-thinning fluid is less than that of Newtonian fluid. Whereas Hayat et al. ${ }^{13)}$ and Haroun ${ }^{14)}$ discussed peristalsis of third order fluid in circular cylindrical tube and asymmetric channel, respectively. The peristaltic flow of non-Newtonian fluid second order fluid was discussed by Elmaboud and Mekheimer. ${ }^{15)}$ A generalization of second and third order fluid is a fourth order fluid. This fluid model has been utilized by Haroun ${ }^{16)}$ to study peristaltic flow in an inclined asymmetric channel.

The shear-thinning/thickening and yield stress effects can also be predicted by making use of generalized Newtonian fluid (GNF) models. The most popular GNF models include power-law model, Carreau model, Sisko model, HerschelBulkley model, Ellis model etc. The use of these models in peristalsis was made by Mishra and $\mathrm{Rao}^{17)}$, Ali and Hayat ${ }^{18)}$, Wang et al. ${ }^{19)}$ and Vajravelu et al.. ${ }^{20)}$ More recently Ali et al. ${ }^{21)}$ reported a semi-analytical solution for peristalsis of Ellis fluid in a planar channel. The effects suspended particles on peristaltic flows were studied by making use of polar fluid models. In such fluid models, the stress tensor is not symmetric. Examples of polar fluid models include couple stress, micropolar, dipolar and micromorphic models. The use of couple stress model to study peristaltic flows was made by Srivastava $^{22)}$, Mekheimer ${ }^{23)}$ and Ali et al.. ${ }^{24)}$ Srinivasacharya et al. ${ }^{25)}$ utilized constitutive equations of micropolar model to investigate peristaltic pumping in a tube.

As discussed above, peristaltic pumping is a fundamental method of transport in many biological processes. In some of these biological settings, fluid may be viscoelastic. The peristaltic flow of viscoelastic fluid models has also been addressed in the past few decades. Mention may be made of studies carried out by Hayat et al. ${ }^{26,27)}$, Wang et al. ${ }^{28)}$, Ali et al. ${ }^{29,30)}$ and Tripathi and Bég. ${ }^{31)}$ In these studies, the authors utilized the constitutive equations of Oldroyd-B model, Johnson-Segalman model and fractional secondgrade model. However, it is found that few attempts are available in the literature dealing with peristaltic flows of Giesekus and FENE-P model despite their effectiveness in predicting a wide variety of polymeric materials. Peristaltic flow of FENE-P fluid has been recently analyzed by Ali and Asghar. $^{32)}$ The Giesekus model introduced by Giesekus ${ }^{33,34)}$ is based on the concept of a deformation dependent tensorial mobility of dissolved molecules. It gives a better description of a polymeric solution and melts. Giesekus fluid enables a qualitative description of a number of well- known properties of viscoelastic fluids, namely shear thinning, non-zero second normal stress coefficient and stress overshoot in transient shear flows. In addition to Weissenberg number, this model was characterized by a dimensionless parameter, known as mobility factor. In investigations carried out by Bird et al. ${ }^{35)}$ and Schleiniger and Weinacht ${ }^{36}$, it was noted that realistic behavior is usually observed for values of mobility factor in between 0 and 0.5 . The peristaltic flow of Giesekus fluid was discussed by Ali and Javed. ${ }^{37)}$ The flow was considered in a planar channel and the flow equations were simplified under the assumptions of long wavelength and low Reynolds number. Recently, Kalantari et al. ${ }^{38)}$ investigated peristaltic flow of a Giesekus fluid in a curved channel for negligible inertia and streamlines curvature effects. Keeping in view the importance of non-Newtonian fluids and tubular structures of biological systems, we propose to analyze the problem of peristaltic flow of Giesekus fluid in a tube. The governing equations have been simplified under the approximations of large wave length and low Reynolds number. ${ }^{3,4,11-13,16)}$ The effects of Weissenberg number and mobility parameter are analyzed on velocity profile, pressure rise per wavelength and trapping phenomenon. A comparison of solution for axisymmetric case is made with the corresponding solution of plane channel.

\section{GOVERNING EQUATIONS}

The flow is assumed to be incompressible; therefore the laws of conservation of mass and momentum take the following form

$$
\begin{aligned}
& \operatorname{div} \overline{\mathbf{V}}=0, \\
& \rho \frac{d \overline{\mathbf{V}}}{d t}=-\nabla \bar{p}+\operatorname{div} \overline{\mathbf{S}},
\end{aligned}
$$

where $\overline{\mathbf{V}}$ is the velocity, $\rho$ the density, $d / d \bar{t}$ the material derivative, $\bar{p}$ the pressure, and $\overline{\mathbf{S}}$ the extra stress tensor. The 
extra stress tensor for Giesekus fluid satisfies the following equation $^{33,34,37)}$

$$
\overline{\mathbf{S}}+\frac{\alpha \bar{\lambda}}{\mu}(\overline{\mathbf{S}}: \overline{\mathbf{S}})+\bar{\lambda} \frac{D \overline{\mathbf{S}}}{D \bar{t}}=\mu \overline{\mathbf{A}}_{1},
$$

in which $\alpha$ and $\bar{\lambda}$ are model parameters representing zeroshear viscosity and zero-shear relaxation time, respectively ${ }^{32}$, $\overline{\mathbf{A}}_{1}$ is the first Rivilin-Ericksen tensor defined by

$$
\overline{\mathbf{A}}_{1}=\overline{\mathbf{L}}+\overline{\mathbf{L}}^{T},
$$

where $\overline{\mathbf{L}}$ is the velocity gradient and

$$
\frac{D \overline{\mathbf{S}}}{D \bar{t}}=\frac{d \overline{\mathbf{S}}}{d \bar{t}}-\overline{\mathbf{L}} \cdot \overline{\mathbf{S}}-\overline{\mathbf{S}} \cdot \overline{\mathbf{L}}^{T}
$$

is the upper convected time derivative. The parameter $\alpha$ appearing in Eq. (3) is another model parameter, and according to Bird et al. ${ }^{33)}$, the term consisting of $\alpha$ is due to anisotropic hydrodynamic drag on the constituent polymer molecules. It is also known in literature as the dimensionless Giesekus-model mobility factor and controls the extensional viscosity and the ratio of second normal stress difference to the first one. For $\alpha=0$ the model (given in (3)) becomes the isotropic upper convected Maxwell (UCM) model, while for $\alpha=1$ the model is simply an anisotropic drag, and for $\alpha>0$ the model represents shear-thinning behavior. For $\alpha=\bar{\lambda}=0$, the model (3) reduces to Newtonian model. The Giesekus model predicts the tension-thickening region for elongational flow, after which a plateau is reached; but it shows the existence of a tension-thinning region at high strain rates. In more practical terms, it describes how the relaxation time of polymer macromolecules is altered when the surrounding molecules are oriented in the flow direction. As $\alpha$ takes non-zero values in the range of $0<\alpha<0.5$, the model can predict a shear-thinning behavior which (in the absence of a solvent viscosity) corresponds to a viscosity which varies proportionally to the inverse of the shear rate. It also predicts a positive first normal stress difference, and a negative second normal stress coefficient with their ratio close to what is found experimentally for polymer melts and concentrated polymer solutions. Unlike the UCM model, the Giesekus model correctly predicts a bounded elongational viscosity and stress overshoot in start-up flows to its credentials. In view of Giesekus ${ }^{33,34)}, \alpha$ must be in the range $0 \leq \alpha \leq 1$. However, Bird et al. ${ }^{35)}$ suggest that for realistic properties $\alpha$ should be such that $0 \leq \alpha \leq 0.5$. The above facts are also delineated by Pourjafar and Sadeghy. ${ }^{39)}$

\section{DEVELOPMENT OF FLOW PROBLEM}

We assume a circular cylindrical tube of radius $a$ filled with an incompressible homogenous Giesekus fluid. The fluid is set into motion by infinite wave trains propagating with speed $c$ along the surface of the tube. A schematic diagram of the flow geometry is illustrated in Fig.1. We employ cylindrical coordinate $(\bar{R}, \bar{Z})$ with $\bar{R}$ along radial direction and $\bar{Z}$ along axial direction to analyze this flow. The geometry of the tube surface is defined through the equation

$$
\bar{h}(\bar{Z}, \bar{t})=a+b \cos \left[\frac{2 \pi}{\lambda^{*}}(\bar{Z}-c \bar{t})\right] .
$$

In Eq.(6), $b$ is the wave amplitude, $\lambda^{*}$ the wavelength, and $\bar{t}$ the time. The appropriate velocity field for the present flow problem is

$$
\overline{\mathbf{V}}=[\bar{U}(\bar{Z}, \bar{R}, \bar{t}), 0, \bar{W}(\bar{Z}, \bar{R}, \bar{t})],
$$

in which $\bar{U}$ and $\bar{W}$ are the radial and axial velocity components, respectively. With the above definition of velocity field, Eqs. (1)-(5) give

$$
\frac{1}{\bar{R}} \frac{\partial}{\partial \bar{R}}(\bar{R} \bar{U})+\frac{\partial \bar{W}}{\partial \bar{Z}}=0,
$$

$\bar{R}$ - component

$$
\begin{aligned}
& \rho\left(\frac{\partial}{\partial \bar{t}}+\bar{U} \frac{\partial}{\partial \bar{R}}+\bar{W} \frac{\partial}{\partial \bar{Z}}\right) \bar{U} \\
& =-\frac{\partial \bar{p}}{\partial \bar{R}}+\frac{1}{\bar{R}} \frac{\partial}{\partial \bar{R}}\left(\bar{R} \bar{S}_{\bar{R} \bar{R}}\right)+\frac{\partial \bar{S} \bar{R} \bar{Z}}{\partial \bar{Z}}-\frac{\bar{S} \overline{\theta \bar{\theta}}}{\bar{R}},
\end{aligned}
$$

$\bar{Z}$ - component

$$
\begin{aligned}
& \rho\left(\frac{\partial}{\partial \bar{t}}+\bar{U} \frac{\partial}{\partial \bar{R}}+\bar{W} \frac{\partial}{\partial \bar{Z}}\right) \bar{W} \\
& =-\frac{\partial \bar{p}}{\partial \bar{Z}}+\frac{\partial \bar{S} \bar{Z} \bar{Z}}{\partial \bar{Z}}+\frac{1}{\bar{R}} \frac{\partial}{\partial \bar{R}}\left(\bar{R} \bar{S}_{\bar{R} \bar{Z}}\right), \\
& \bar{S} \bar{R} \bar{R}+\frac{\alpha \bar{\lambda}}{\mu}\left(\bar{S}_{\bar{R}}^{2}+\bar{S}_{\bar{R} \bar{\theta}}^{2}+\bar{S}_{\bar{R} \bar{Z}}^{2}\right) \\
& +\bar{\lambda}\left[\begin{array}{l}
\left(\frac{\partial}{\partial \bar{t}}+\bar{U} \frac{\partial}{\partial \bar{R}}+\bar{W} \frac{\partial}{\partial \bar{Z}}\right) \bar{S}_{\bar{R} \bar{R}} \\
-2 \frac{\partial \bar{U}}{\partial \bar{R}} \bar{S}_{\bar{R}} \bar{R}+2 \frac{\partial \bar{U}}{\partial \bar{Z}} \bar{S}_{\bar{R}} \bar{Z}
\end{array}\right]=2 \mu \frac{\partial \bar{U}}{\partial \bar{R}},
\end{aligned}
$$

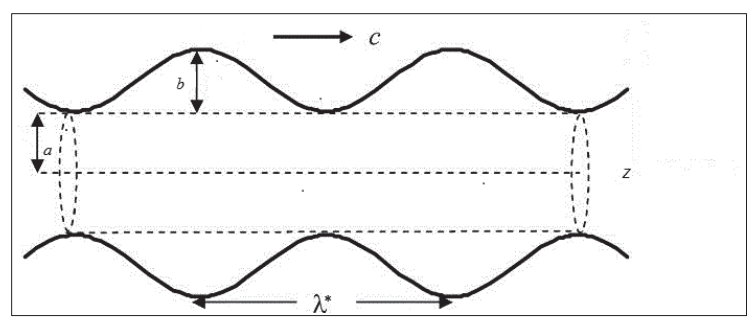

Fig. 1. Schematic diagram of flow geometry. 


$$
\begin{aligned}
& \bar{S}_{\bar{Z} \bar{R}}+\frac{\alpha \bar{\lambda}}{\mu}\left(\left(\bar{S}_{\bar{R} \bar{R}}+\bar{S}_{\bar{\theta} \bar{\theta}}\right) \bar{S}_{\bar{R} \bar{\theta}}+\bar{S}_{\bar{R} \bar{Z}} \bar{S}_{\bar{Z} \bar{\theta}}\right) \\
& +\bar{\lambda}\left[\begin{array}{c}
\left(\frac{\partial}{\partial t}+\bar{U} \frac{\partial}{\partial \bar{R}}+\bar{W} \frac{\partial}{\partial \bar{Z}}\right) \bar{S} \bar{R} \bar{Z} \\
-\left(\frac{\partial \bar{U}}{\partial \bar{R}}+\frac{\partial \bar{W}}{\partial \bar{Z}}\right) \bar{S} \bar{R} \bar{Z} \\
+\frac{\partial \bar{U}}{\partial \bar{Z}} \bar{S} \bar{Z} \bar{Z}-\left(\bar{S} \bar{R} \bar{R} \frac{\partial \bar{W}}{\partial \bar{R}}\right)
\end{array}\right]=\mu\left(\frac{\partial \bar{W}}{\partial \bar{R}}+\frac{\partial \bar{U}}{\partial \bar{Z}}\right), \\
& \bar{S}_{\bar{Z} \bar{Z}}+\frac{\alpha \bar{\lambda}}{\mu}\left(\bar{S}_{\bar{Z}}^{2} \bar{R}+\bar{S}_{\bar{Z}}^{2} \bar{\theta}+\bar{S}_{\bar{Z}}^{2} \bar{Z}\right) \\
& +\bar{\lambda}\left[\begin{array}{c}
\left(\frac{\partial}{\partial t}+\bar{U} \frac{\partial}{\partial \bar{R}}+\bar{W} \frac{\partial}{\frac{\partial}{\partial \bar{Z}}}\right) \bar{S} \overline{\bar{Z}} \bar{Z} \\
-\left(\frac{\partial \bar{W}}{\partial \bar{R}} \bar{S} \bar{Z} \bar{Z}+\frac{\partial \bar{W}}{\partial \bar{Z}} \bar{Z} \bar{Z}\right) \\
-\left(\bar{S} \bar{Z} \bar{R} \frac{\partial \bar{W}}{\partial \bar{R}}+\bar{S} \bar{Z} \bar{Z} \frac{\partial \bar{W}}{\partial \bar{Z}}\right)
\end{array}\right]=2 \mu \frac{\partial \bar{W}}{\partial \bar{Z}}, \\
& \bar{S}_{\bar{R} \bar{\theta}}+\frac{\alpha \bar{\lambda}}{\mu}\left(\left(\bar{S}_{\bar{R} \bar{R}}+\bar{S}_{\bar{\theta}} \bar{\theta}\right) \bar{S}_{\bar{R} \bar{\theta}}+\bar{S}_{\bar{R} \bar{Z}} \bar{S} \bar{Z} \bar{\theta}\right)
\end{aligned}
$$

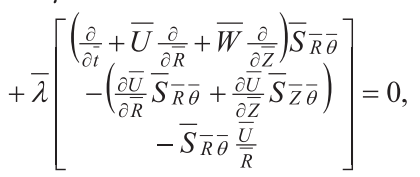

$$
\begin{aligned}
& \bar{S}_{\bar{\theta} \bar{\theta}}+\frac{\alpha \bar{\lambda}}{\mu}\left(\bar{S}_{\bar{\theta} \bar{R}}^{2}+\bar{S}_{\bar{\theta} \bar{\theta}}^{2}+\bar{S}_{\bar{\theta} \bar{Z}}^{2}\right) \\
& +\bar{\lambda}\left[\begin{array}{l}
\left(\frac{\partial}{\partial \bar{t}}+\bar{U} \frac{\partial}{\partial \bar{R}}+\bar{W} \frac{\partial}{\partial \bar{Z}}\right) \bar{S}_{\bar{\theta} \bar{\theta}} \\
-\bar{S}_{\bar{\theta}} \overline{\bar{R}} \overline{\bar{U}}-\bar{S}_{\bar{\theta}} \overline{\bar{\theta}} \overline{\bar{U}}
\end{array}\right]=2 \mu \frac{\bar{U}}{\bar{R}}, \\
& \bar{S}_{\bar{\theta}} \bar{Z}+\frac{\alpha \bar{\lambda}}{\mu}\left(\bar{S}_{\bar{\theta}} \overline{\bar{R}} \bar{S}_{\bar{R} \bar{Z}}+\bar{S}_{\bar{\theta} \bar{Z}} \bar{S}_{\bar{\theta}} \bar{\theta}+\bar{S}_{\bar{Z} \bar{Z}} \bar{S}_{\bar{Z} \bar{\theta}}\right) \\
& +\bar{\lambda}\left[\begin{array}{l}
\left(\frac{\partial}{\partial \bar{t}}+\bar{U} \frac{\partial}{\partial \bar{R}}+\bar{W} \frac{\partial}{\partial \bar{Z}}\right) \bar{S}_{\bar{\theta}} \bar{Z} \\
-\frac{\bar{U}}{\bar{R}} \bar{S}_{\bar{\theta}} \bar{Z}-\left(\bar{S} \bar{\theta} \bar{R} \frac{\partial \bar{W}}{\partial \bar{R}}+\bar{S}_{\bar{\theta}} \overline{\bar{Z}} \frac{\partial \bar{W}}{\partial \bar{Z}}\right)
\end{array}\right]=0 .
\end{aligned}
$$

The flow problem under consideration is subject to following boundary conditions

$$
\frac{\partial \bar{W}}{\partial \bar{R}}=0 \text {, at } \bar{R}=0 \text {, and } \bar{U}=\frac{\partial \bar{h}}{\partial \bar{t}}, \bar{W}=0 \text { at } \bar{R}=\bar{h} .
$$

The condition at $\bar{R}=0$ represents the symmetry of axial velocity while the conditions at $\bar{R}=\bar{h}$ illustrate the adherence of the fluid at the boundary. For subsequent analysis, we switch from fixed frame to wave frame. These frames are related through the following transformation

$$
\bar{z}=\bar{Z}-c \bar{t}, \bar{r}=\bar{R}, \bar{u}=\bar{U}, \bar{w}=\bar{W}-c,
$$

where $(\bar{u}, \bar{w})$ are the radial and axial velocity components in the wave frame. Let us introduce following dimensionless variables and parameters

$$
z=\frac{2 \pi \bar{z}}{\lambda^{*}}, r=\frac{\bar{r}}{a}, u=\frac{\bar{u}}{c}, w=\frac{\bar{w}}{c}, h=\frac{\bar{h}}{a}, S=\frac{a \bar{S}}{\mu c}, p=\frac{2 \pi a^{2}}{\lambda \mu c} \bar{p} .
$$

By virtue of these parameters, the governing equations can be casted as

$$
\begin{aligned}
& \frac{1}{r} \frac{\partial}{\partial r}(r u)+\delta \frac{\partial w}{\partial z}=0 \\
& \delta \operatorname{Re}\left(w \frac{\partial}{\partial z}+u \frac{\partial}{\partial r}\right) u=-\frac{\partial p}{\partial r}+\delta\left[\frac{\partial S_{r r}}{\partial r}+\frac{S_{r r}}{r}+\delta \frac{\partial S_{z r}}{\partial z}-\frac{S_{\theta \theta}}{r}\right] \\
& \operatorname{Re}\left(\delta w \frac{\partial}{\partial z}+u \frac{\partial}{\partial r}\right) w=-\frac{\partial p}{\partial z}+\left[\frac{\partial S_{r z}}{\partial r}+\frac{S_{r r}}{r}+\delta \frac{S_{z z}}{\partial z}\right]
\end{aligned}
$$$$
S_{r r}+\alpha W e\left(S_{r r}^{2}+S_{r \theta}^{2}+S_{r z}^{2}\right)+W e\left[\begin{array}{c}
\left(u \frac{\partial}{\partial r}+\delta w \frac{\partial}{\partial z}\right) S_{r r}- \\
\left.\frac{\partial u}{\partial r} S_{r r}+\delta \frac{\partial u}{\partial z} S_{r r}\right) \\
-\left(S_{r r} \frac{\partial u}{\partial r}+S_{r z} \frac{\partial u}{\partial r}\right)
\end{array}\right]=2 \frac{\partial u}{\partial r},
$$$$
S_{z z}+\alpha W e\left(S_{z r}^{2}+S_{z \theta}^{2}+S_{z z}^{2}\right)+W e\left[\begin{array}{c}
\left(u \frac{\partial}{\partial r}+\delta w \frac{\partial}{\partial z}\right) S_{z z}- \\
\left(\frac{\partial w}{\partial r} S_{r z}+\delta \frac{\partial w}{\partial z} S_{z z}\right) \\
-\left(S_{z r} \frac{\partial w}{\partial r}+S_{z z} \delta \frac{\partial w}{\partial z}\right)
\end{array}\right]=2 \delta \frac{\partial w}{\partial z},
$$$$
S_{\theta \theta}+\alpha W e\left(S_{\theta r}^{2}+S_{\theta \theta}^{2}+S_{\theta z}^{2}\right)+W e\left[\begin{array}{c}
\left(u \frac{\partial}{\partial r}+\delta w \frac{\partial}{\partial z}\right) S_{\theta \theta}- \\
\frac{2 u}{r} S_{\theta \theta}
\end{array}\right]=2 \frac{u}{r},
$$

$S_{r \theta}+\alpha W e\left(\left(S_{r r}+S_{\theta \theta}\right) S_{r \theta}+S_{r z} S_{z \theta}\right)+W e\left[\begin{array}{c}\left(u \frac{\partial}{\partial r}+\delta w \frac{\partial}{\partial z}\right) S_{r \theta}- \\ \left(\frac{\partial u}{\partial r} S_{r \theta}+\delta \frac{\partial u}{\partial r} S_{z \theta}\right) \\ -S_{r \theta} \frac{u}{r}\end{array}\right]=0$,

$$
\begin{aligned}
& S_{r z}+\alpha W e\left(S_{r r} S_{r z}+S_{r \theta} S_{\theta z}+S_{r z} S_{z z}\right) \\
& +W e\left[\begin{array}{c}
\left(u \frac{\partial}{\partial r}+\delta w \frac{\partial}{\partial z}\right) S_{r z}- \\
\left.\frac{\partial u}{\partial r} S_{r z}+\delta \frac{\partial u}{\partial r} S_{z z}\right) \\
-\left(S_{r r} \frac{\partial w}{\partial r}+\delta S_{r z} \frac{\partial u}{\partial r}\right)
\end{array}\right]=\left(\frac{\partial w}{\partial r}-\delta \frac{\partial u}{\partial r}\right),
\end{aligned}
$$

$S_{z \theta}+\alpha W e\left(S_{z r} S_{r \theta}+S_{z \theta} S_{\theta \theta}+S_{z z} S_{z \theta}\right)+W e\left[\begin{array}{c}\left(u \frac{\partial}{\partial r}+\delta w \frac{\partial}{\partial z}\right) S_{z \theta}- \\ \left.\frac{\partial w}{\partial r} S_{r \theta}+\delta \frac{\partial w}{\partial r} S_{z \theta}\right) \\ -S_{z \theta} \frac{u}{r}\end{array}\right]=0$,

where $\operatorname{Re}=(\rho c a / \mu)$ is the Reynolds number, $\delta=\left(2 \pi a / \lambda^{*}\right)$ is the ratio of the radius of the tube to the wavelength of the peristaltic wave and $W e=\lambda a / c$ Weissenberg number. Introducing the stream function $\psi(r, z)$ by the relations

$$
u=\frac{-\delta}{r} \frac{\partial \psi}{\partial z}, w=\frac{1}{r} \frac{\partial \psi}{\partial r}
$$

the continuity equation is satisfied identically and Eqs. (21-28) under long wavelength and low Reynolds number assumption ${ }^{2,10,19,37)}$ reduce to

$$
\frac{\partial p}{\partial r}=0,
$$

$$
\frac{\partial p}{\partial z}=\frac{1}{r} \frac{\partial\left(r S_{r z}\right)}{\partial r},
$$




$$
\begin{aligned}
& S_{r r}+\alpha W e\left(S_{r r}^{2}+S_{r \theta}^{2}+S_{r z}^{2}\right)=0 \\
& S_{r z}+\alpha W e\left(S_{r r} S_{r z}+S_{r \theta} S_{\theta z}+S_{r z} S_{z z}\right) \\
& \quad-W e\left[S_{r r} \frac{\partial}{\partial r}\left(\frac{1}{r} \frac{\partial \psi}{\partial r}\right)\right]=\frac{\partial}{\partial r}\left(\frac{1}{r} \frac{\partial \psi}{\partial r}\right) \\
& S_{z z}+\alpha W e\left(S_{z r}^{2}+S_{z \theta}^{2}+S_{z z}^{2}\right)-2 W e \frac{\partial}{\partial r}\left(\frac{1}{r} \frac{\partial \psi}{\partial r}\right) S_{r z}=0 \\
& S_{\theta \theta}+\alpha W e\left(S_{\theta r}^{2}+S_{\theta \theta}^{2}+S_{\theta z}^{2}\right)=0 \\
& S_{r \theta}+\alpha W e\left(S_{r r} S_{r \theta}+S_{r \theta} S_{\theta \theta}+S_{r z} S_{z \theta}\right)=0 \\
& S_{z \theta}+\alpha W e\left(S_{z r} S_{r \theta}+S_{z \theta} S_{\theta \theta}+S_{z z} S_{z \theta}\right) \\
& \quad+W e\left(\frac{1}{r^{2}} \frac{\partial \psi}{\partial r}-\frac{1}{r} \frac{\partial^{2} \psi}{\partial r^{2}}\right) S_{z \theta}=0
\end{aligned}
$$

Because of the invariance of the flow with respect to $\theta$ we expect no momentum flux in the $\theta$-direction, so we set $S_{r \theta}=S_{z \theta}=0$, as the consequence of this argument $S_{\theta \theta}=0$ also turns out to be zero. In view of this argument, Eqs. (35-37) can be reduced to

$$
\begin{aligned}
& S_{r r}+\alpha W e\left(S_{r r}^{2}+S_{r z}^{2}\right)=0 \\
& S_{r z}+\alpha W e S_{r z}\left(S_{r r}+S_{z z}\right)-W e\left[S_{r r} \frac{\partial}{\partial r}\left(\frac{1}{r} \frac{\partial \psi}{\partial r}\right)\right]=\frac{\partial}{\partial r}\left(\frac{1}{r} \frac{\partial \psi}{\partial r}\right) \\
& S_{z z}+\left(S_{z r}^{2}+S_{z z}^{2}\right)-2 W e \frac{\partial}{\partial r}\left(\frac{1}{r} \frac{\partial \psi}{\partial r}\right) S_{r z}=0
\end{aligned}
$$

The boundary conditions (17) in term stream function read

$$
\begin{aligned}
& \psi=0, \frac{\partial}{\partial r}\left(\frac{1}{r} \frac{\partial \psi}{\partial r}\right)=0 \text { at } r=0, \psi=F, \\
& \frac{1}{r} \frac{\partial \psi}{\partial r}=-1, \text { at } r=h=1+\phi \cos z,
\end{aligned}
$$

The additional boundary conditions $\psi(0)=0$ and $\psi(h)=F$ are the consequence of the prescription of constant flow rate at each cross-section. ${ }^{14)}$ These conditions are necessary to obtain a unique solution of the problem. The dimensionless mean flow rate $\Theta$ in fixed frame and $F$ in wave frame are related through the following expression ${ }^{14}$

$$
\Theta=F+\frac{1}{2}\left(1+\frac{\phi^{2}}{2}\right) \text {. }
$$

The dimensionless pressure rise over one wavelength can be calculated by the following expression

$$
\Delta p=\int_{0}^{2 \pi} \frac{d p}{d z} d z
$$

\section{SOLUTION OF THE PROBLEM}

Eq. (31) implies that $p \neq p(r)$ therefore $\partial p / \partial z$ in Eq. (31) can be replaced with $d p / d z$. Integrating Eq. (31) and using the second boundary condition in (42), we can write

$$
S_{r z}=\frac{d p}{d z} \frac{r}{2}
$$

From Eqs. (38) and (39), we arrive at

$$
\begin{aligned}
& S_{r r}=\frac{-1 \pm \sqrt{1-4 \alpha^{2} W e^{2} S_{r z}^{2}}}{2 \alpha W e}, \\
& S_{z z}=\frac{\left(1+W e S_{r r}\right) \frac{\partial}{\partial r}\left(\frac{1}{r} \frac{\partial \psi}{\partial r}\right)}{\alpha W e S_{r z}}-\frac{1+\alpha W e S_{r r}}{\alpha W e},
\end{aligned}
$$

For further analysis, we proceed with positive sign in Eq. (45). ${ }^{37)}$ Inserting expressions (45) and (46) into Eq. (39), we get following equation in term of stream function $\psi$

$$
\frac{\partial}{\partial r}\left(\frac{1}{r} \frac{\partial \psi}{\partial r}\right)=\frac{1+a_{1} d_{1}\left\{\left(-1+\sqrt{1-b_{1}\left(\frac{d p}{d z}\right)^{2} r^{2}}\right)\right\}}{\left(1+\left\{a_{1}\left(-1+\sqrt{1-b_{1}\left(\frac{d p}{d z}\right)^{2} r^{2}}\right)\right\}\right)^{2}} \frac{d p}{d z} \frac{r}{2},
$$

where $d_{1}=1 / 2 \alpha, b_{1}=\alpha^{2} W e^{2}$ and $a_{1}=1-d_{1}$. The solution of the Eq. (47) subject to the boundary conditions $\psi=0, \frac{\partial}{\partial r}\left(\frac{1}{r} \frac{\partial \psi}{\partial r}\right)=0$ at $r=0$ is

$$
\begin{aligned}
\psi= & \frac{1}{24 b_{1}^{2}\left(\frac{d p}{d z}\right)^{3} d_{1}^{5}}\left[-4 a_{1} d_{1}^{3}+6\left(-1+d_{1}\right) d_{1}\left(a_{1}\left(-4+d_{1}\right)+3 d_{1}\right)\right. \\
& +3 d_{1}^{2}\left(d_{1}+4 b_{1} \frac{d p}{d z} c_{1} d_{1}^{3}+a_{1}\left(-4+3 d_{1}\right)\right) \\
& -6\left(-1+d_{1}\right) d_{1}\left(a_{1}\left(-4+d_{1}\right)+3 d_{1}\right) \sqrt{1-b_{1}\left(\frac{d p}{d z}\right)^{2} r^{2}} \\
& \left.+4 a_{1} d_{1}^{3}\left(1-b_{1}\left(\frac{d p}{d z}\right)^{2} r^{2}\right)^{\frac{3}{2}}\right) 3 d_{1}^{2}\left(d_{1}+4 b_{1}\left(\frac{d p}{d z}\right)^{2} c_{1} d_{1}^{3}\right. \\
& \left.+a_{1}\left(-4+3 d_{1}\right)\left(-1+b_{1}\left(\frac{d p}{d z}\right)^{2} r^{2}\right)-6\left(-1+d_{1}\right)^{2}\left(a_{1}\left(-4+d_{1}\right)+3 d_{1}\right) \log \right) \\
& {\left[1+d_{1}\left(-1+d_{1}\left(-1+\sqrt{1-b_{1}\left(\frac{d p}{d z}\right)^{2} r^{2}}\right)\right)\right]-6 d_{1}^{2}\left(a_{1}\left(-2+d_{1}\right)+d_{1}\right) } \\
& \left.\left.+\left(-1+b_{1}\left(\frac{d p}{d z}\right)^{2} r^{2}\right) \log \left[1+d_{1}\left(-1+\sqrt{1-b_{1}\left(\frac{d p}{d z}\right)^{2} r^{2}}\right)\right]\right)\right],
\end{aligned}
$$

In view of boundary condition $\psi(h)=F$ we get the following nonlinear equation in $d p / d z$ 


$$
\begin{aligned}
F= & \frac{1}{24 b_{1}^{2} c^{3} d_{1}^{5}}\left[-4 a_{1} d_{1}^{3}+6\left(-1+d_{1}\right) d_{1}\left(a_{1}\left(-4+d_{1}\right)+3 d_{1}\right)\right. \\
& +3 d_{1}^{2}\left(d_{1}+4 b_{1} \frac{d p}{d z} d_{1}^{3}+a_{1}\left(-4+3 d_{1}\right)\right) \\
& -6\left(-1+d_{1}\right) d_{1}\left(a_{1}\left(-4+d_{1}\right)+3 d_{1}\right) \sqrt{1-b_{1}\left(\frac{d p}{d z}\right)^{2} h^{2}} \\
& \left.+4 a_{1} d_{1}^{3}\left(1-b_{1}\left(\frac{d p}{d z}\right)^{2} h^{2}\right)^{\frac{3}{2}}\right) 3 d_{1}^{2}\left(d_{1}+4 b_{1}\left(\frac{d p}{d z}\right)^{2} d_{1}^{3}\right. \\
& \left.+a_{1}\left(-4+3 d_{1}\right)\left(-1+b_{1}\left(\frac{d p}{d z}\right)^{2} h^{2}\right)-6\left(-1+d_{1}\right)^{2}\left(a_{1}\left(-4+d_{1}\right)+3 d_{1}\right) \log \right) \\
& {\left[1+d_{1}\left(-1+d_{1}\left(-1+\sqrt{1-b_{1}\left(\frac{d p}{d z}\right)^{2} h^{2}}\right)\right)\right]-6 d_{1}^{2}\left(a_{1}\left(-2+d_{1}\right)+d_{1}\right) } \\
& \left.\left.+\left(-1+b_{1}\left(\frac{d p}{d z}\right)^{2} h^{2}\right) \log \left[1+d_{1}\left(-1+\sqrt{1-b_{1}\left(\frac{d p}{d z}\right)^{2} h^{2}}\right)\right]\right)\right],
\end{aligned}
$$

Using the symbolic software Mathematica 8, the above equation is solved for unknown constant $d p / d z$ at each crosssection $z$ for a given set of parameters. Thus, solution for a given set of parameters is completely known at each crosssection $z$. To find an approximate solution of Eq. (47), we expand the radical term using binomial expansion up to two terms and write

$$
\frac{\partial}{\partial r}\left(\frac{1}{r} \frac{\partial \psi}{\partial r}\right)=\left(\frac{1-a_{2}\left(\frac{d p}{d z}\right)^{2} r^{2}}{\left(1-b_{1}\left(\frac{d p}{d z}\right)^{2} r^{2}\right)^{2}}\right) \frac{d p}{d z} \frac{r}{2},
$$

where $a_{2}=\alpha(2 \alpha-1) W e^{2}$. Twice integration of Eq. (50) and utilization of boundary conditions $\psi(0)=0$ and $\left.\frac{1}{r} \frac{\partial \psi}{\partial r}\right|_{r=h}=-1$ yield

$$
\begin{aligned}
\psi= & \frac{1}{8 b_{2}^{2}\left(\frac{d p}{d z}\right)^{3}\left(-1+b_{1}\left(\frac{d p}{d z}\right)^{2} h^{2}\right)} \\
& {\left[\begin{array}{c}
\left(b_{2}+4 b_{1}^{3}\left(\frac{d p}{d z}\right)^{2} h^{2}+a_{2}\left(-2+b_{1}\left(\frac{d p}{d z}\right)^{2} h^{2}\right)\right)\left(-2+b_{1}\left(\frac{d p}{d z}\right)^{2} r^{2}\right) \\
a_{2}\left(1+b_{1}^{2}\left(\frac{d p}{d z}\right)^{4} h^{2} r^{2}-b_{1}\left(\frac{d p}{d z}\right)^{2}\left(h^{2}+r^{2}\right) \log \left[-1+b_{1}\left(\frac{d p}{d z}\right)^{2} h^{2}\right]\right. \\
-\left(-1+b_{1}\left(\frac{d p}{d z}\right)^{2} h^{2}\right)\left(b_{1}+a_{2}\left(-2+b_{1}\left(\frac{d p}{d z}\right)^{4} r^{2}\right)\right) \log \left[-1+b_{1}\left(\frac{d p}{d z}\right)^{2} r^{2}\right]
\end{array}\right.}
\end{aligned}
$$

The constant $d p / d z$ at each cross-section can be obtained by using the boundary condition $\psi=F$ at $r=h$, and solving the following nonlinear algebraic equation

$$
\begin{aligned}
F= & \frac{1}{8 b_{2}^{2}\left(\frac{d p}{d z}\right)^{3}\left(-1+b_{1}\left(\frac{d p}{d z}\right)^{2} h^{2}\right)} \\
& {\left[\begin{array}{c}
\left(b_{1}+4 b_{1}^{3}\left(\frac{d p}{d z}\right)^{2} h^{2}+a_{2}\left(-2+b_{1}\left(\frac{d p}{d z}\right)^{2} h^{2}\right)\right)\left(-2+b_{1}\left(\frac{d p}{d z}\right)^{2} h^{2}\right) \\
a_{2}\left(1+b_{1}^{2}\left(\frac{d p}{d z}\right)^{4} h^{4}-b_{1}\left(\frac{d p}{d z}\right)^{2}\left(2 h^{2}\right) \log \left[-1+b_{1}\left(\frac{d p}{d z}\right)^{2} h^{2}\right]\right. \\
-\left(-1+b_{1}\left(\frac{d p}{d z}\right)^{2} h^{2}\right)\left(b_{1}+a_{2}\left(-2+b_{1}\left(\frac{d p}{d z}\right)^{4} h^{2}\right)\right) \log \left[-1+b_{1}\left(\frac{d p}{d z}\right)^{2} h^{2}\right]
\end{array}\right.}
\end{aligned}
$$

In the next section we shall first give the comparison of approximate and exact solution of Eq. (47) and then discuss the effects of various emerging parameter on flow characteristics, pumping and trapping phenomena.

\section{RESULTS AND DISCUSSION}

First, we present a comparison of exact and approximate solution of Eq. (51). Fig. 2 is plotted to serve the purpose. This figure shows that for $\alpha=0.5$ and $W e=0.3$ both solutions are in excellent agreement. By increasing the value of We to 1.5 , approximate solution slightly deviates from the exact solution. A further deviation is observed when $W e$ is increased to 2 . In this case the deviation is prominent in the vicinity of the center of the tube.

However, the approximate solution shows deviation from the exact solution over the whole cross-section when we take the value of $W e$ equal to 3 . Thus it is concluded that when $\phi=0.4$ and $F=-0.2$, range of validity of approximate solution is $0 \leq \alpha \leq 0.5$ and $0 \leq W e \leq 2$. Similar ranges of parameters of the Giesekus model can be identified for other values of $\phi$ and $F$.

The variation of axial velocity $w(r)$ at a cross-section $z=\pi$ for different values of $W e$ and $\alpha$ is shown in Fig. 3. This figure shows that the magnitude of velocity decrease at the center of the channel by increasing both $\alpha$ and We. Further, a flattening trend is observed in the velocity profile for large values of both $\alpha$ and $W e$. This is not surprising realizing the fact that Giesekus model predicts shear-thinning behavior for values of $\alpha$ in the range $0 \leq \alpha \leq 0.5$.

This trend in velocity indicates the existence of thin boundary layer at the tube wall. In such case, the largest gradients in velocity are confined in a thin layer in the vicinity of the tube wall and the fluid outside this layer moves with a uniform velocity. Such a non-trivial behavior of velocity cannot be predicted using the constitutive equations of Maxwell and Newtonian models which do not include the mobility parameter $\alpha$. It is interesting to note that such effects of $\alpha$ and $W e$ on velocity profile are not visible for channel flow. ${ }^{37)}$ The velocity profiles corresponding to tube and channel flow are compared in Fig. 4 for different values of $\alpha$ and $W e$. It is observed that for similar choice of Giesekus fluid parameters, the magnitude of velocity for tube flow is higher than the magnitude of velocity in a plane channel.

The relationship between pressure rise per wavelength and flow rate for various values of $\alpha$ and $W e$ is shown through Fig. 5. We note that pressure rise per wavelength decreases in peristaltic pumping region $(\Delta p>0, \Theta>0)$ by increasing either $\alpha$ or $W e$ for a fixed value of prescribed flow rate. This is not 

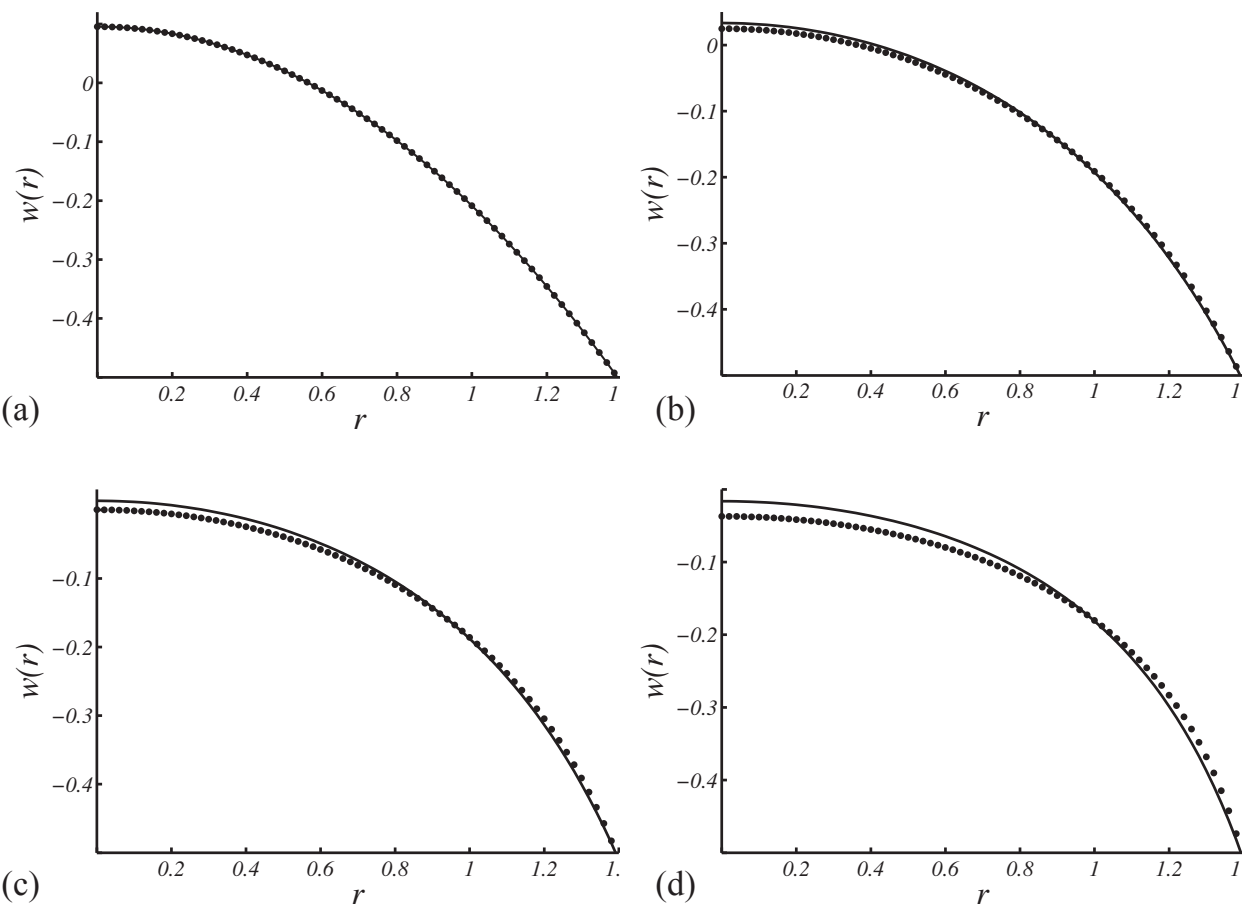

Fig. 2. Plots showing the comparison of approximate (solid line) and exact solution (dotted line) for $F=-0.2$, $\phi=0.4$ (a) $\alpha=0.5, W e=0.3$ (b) $\alpha=0.5, W e=1.5$ (c) $\alpha=0.5, W e=2$ (d) $\alpha=0.5, W e=3$.
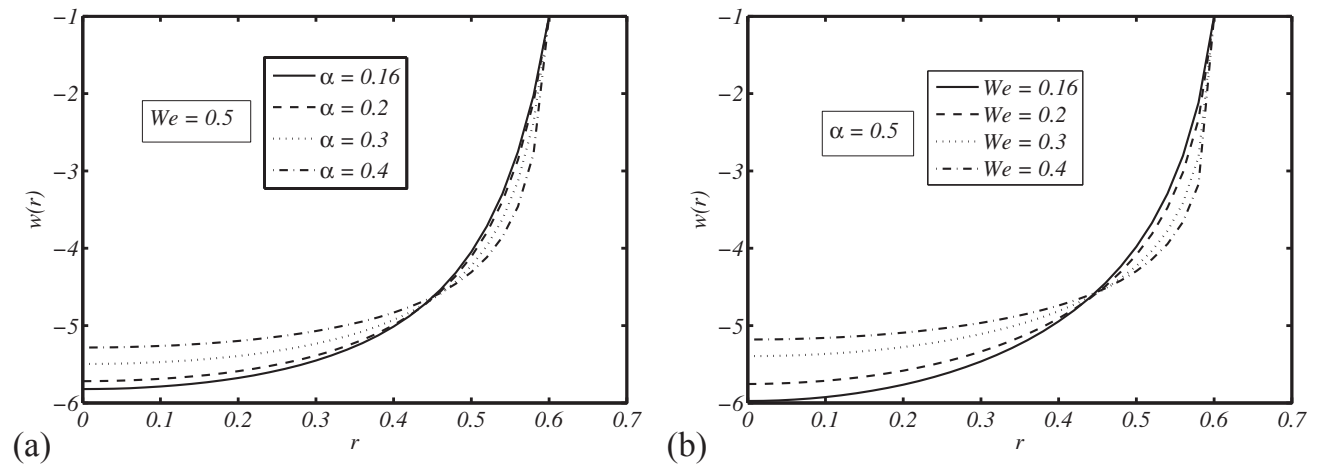

Fig. 3. Plots showing $w(r)$ at cross-section $z=\pi$ for different values of (a) $\alpha(W e=0.5)$ and (b) $W e(\alpha=0.5)$. The other parameters are $F=0.8$ and $\phi=0.4$.
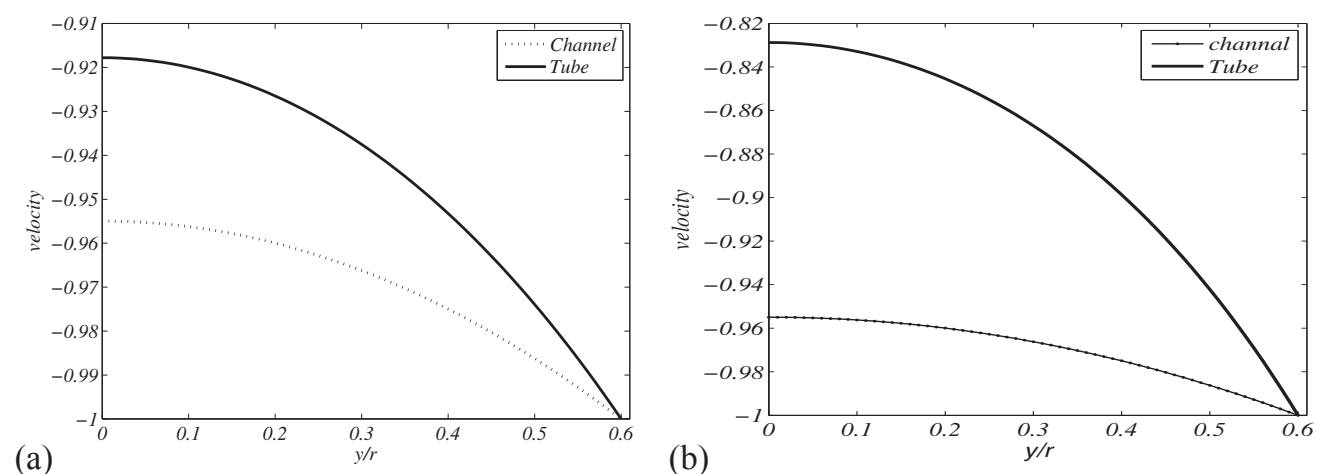

Fig. 4. Plots showing the comparison of velocity in tube and channel when $F=-0.25, \phi=0.4$ and $z=x=\pi$. (a) for $\alpha=0.1$ when $W e=0.5$ (b) for $W e=0.5$ when $\alpha=0.3$. 
surprising realizing the fact that by an increase in the mobility factor or Weissenberg number, shear-thinning starts at lower shear rates so that the gap inside the tube is now occupied by a low-viscosity fluid. Obviously, low-viscosity fluid elements present less resistance to the peristaltic waves. The free pumping flux i.e., $\Theta$ for $\Delta p=0$ is found to be independent of both $\alpha$ and We. The plots in Fig. 5 further reveal an increase in $\Delta p$ by increasing $\alpha$ and $W e$ in augmented pumping region $(\Delta p<0, \Theta<0)$. Fig. 6 presents a comparison of pressure rise per wavelength between tube and channel flow of Giesekus fluid. This figure illustrates that pressure rise per wavelength $(\Delta p)$ for axisymmetric case achieves higher values than the pressure rise per wavelength for the plane channel geometry.

The streamlines patterns for different values of $\alpha$ and $W e$ are shown in Fig.7. This figure illustrates that the size and circulation of trapped bolus decrease by increasing either $\alpha$ or We. However, this decrease is rapid when $\alpha$ is kept fixed and $W e$ is increased. As an implication of this observation, a much greater mixing is realized in fluids with lower values of mobility factor (shear-thickening fluids). A comparison of streamlines for channel and tube geometry for different values of $\alpha$ and $W e$ is carried out in Figs. 8 and 9, respectively. For same choice of $\alpha$ and $W e$, the size of trapped bolus in plane channel is bigger than the size of trapped bolus in plane circular tube. In this way, a much greater mixing of fluid is realized in a plane channel than in a circular tube for a Giesekus fluid.

\section{CONCLUSIONS}

Axisymmetric peristaltic flow of Giesekus fluid is dealt using a semi-analytic approach under long wavelength and low Reynolds number assumptions. The effects of pertinent parameter of Giesekus fluid on various features of peristaltic motion are discussed in detailed. The graphical results for a symmetric case are also compared with corresponding results
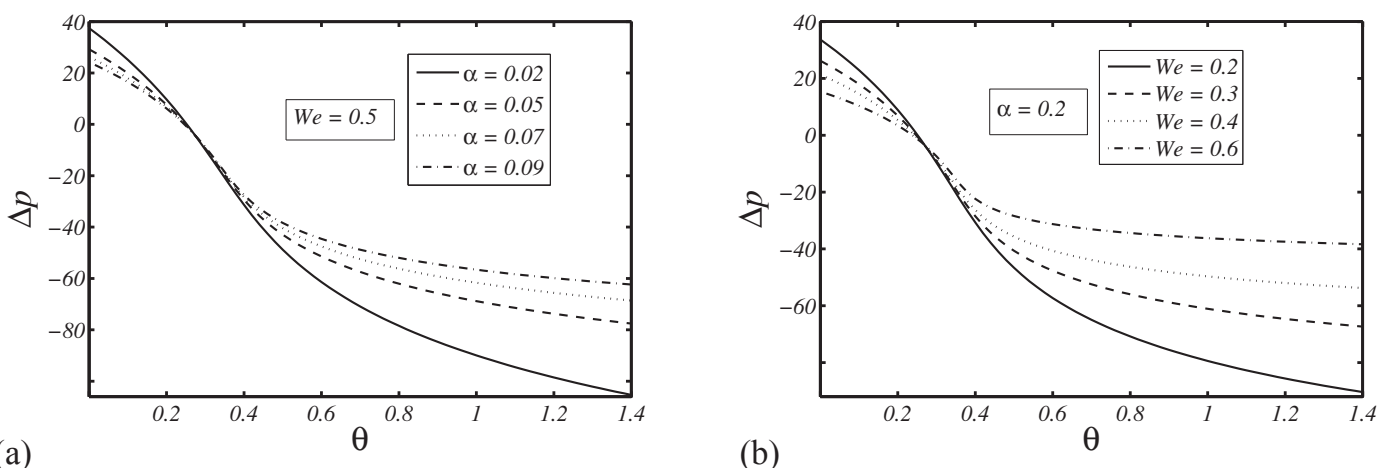

Fig. 5. Plots showing $\Delta p$ versus $\Theta$ for different values of (a) $\alpha$ (We=0.5) and (b) $W e(\alpha=0.2)$ with $\phi=0.4$.
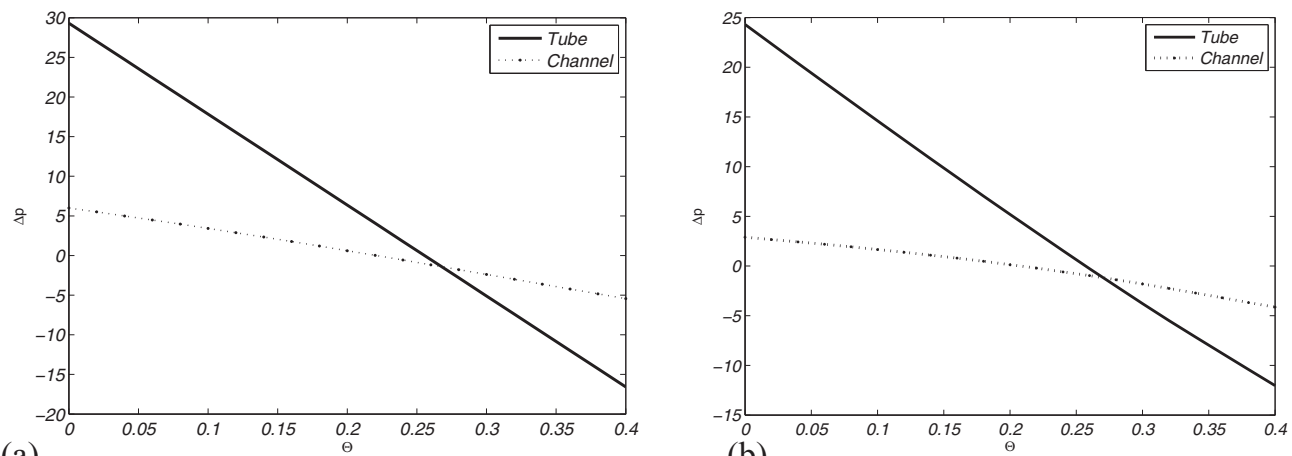

(a)

(b)

Fig.6. Plots showing the comparison of pressure rise in tube and channel when $\phi=0.4$ (a) for $\alpha=0.5$ when $W e=0.2$ (b) for $W e=0.2$ when $\alpha=0.3$. 
of planner channel.

The important findings of the present study are;

1. The velocity profile in axisymmetric case exhibits boundary layer character with increasing $W e$ and $\alpha$. Such a character of velocity is not evident in the planner channel for a similar choice of parameters.

2. The magnitude of axial velocity in tube center shows decreasing trend with increasing both $\alpha$ and $W e$.

3. The pressure rise per wavelength decreases when the flow geometry changes from a symmetric tube to a plane channel.

4. The Giesekus model predicts lower values of pressure rise in the pumping region than the classical Newtonian and Maxwell models.
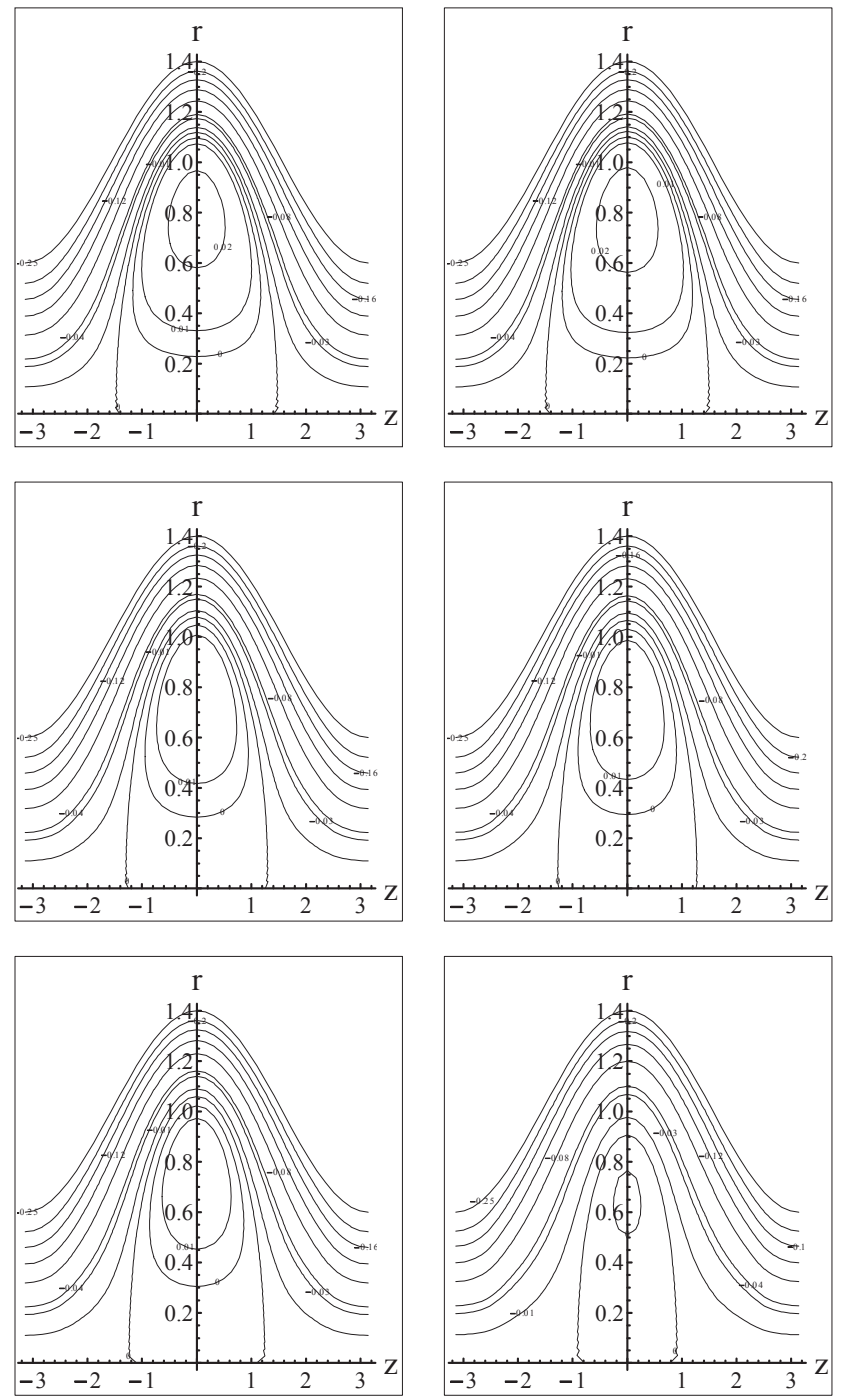

Fig.7. Streamline for $(\alpha=0.05,0.3,0.4), W e=1$ (left panels) and for (We $=0.3,0.8,1.5), \alpha=0.5$ (Right panels). The other parameters are $F=-0.25$ and $\phi=0.4$.
5. The size and circulation of the trapped bolus decreases in going from Newtonian to Giesekus fluid.

6. The size and circulation of trapped bolus is greater in a plane channel than in a circular tube.
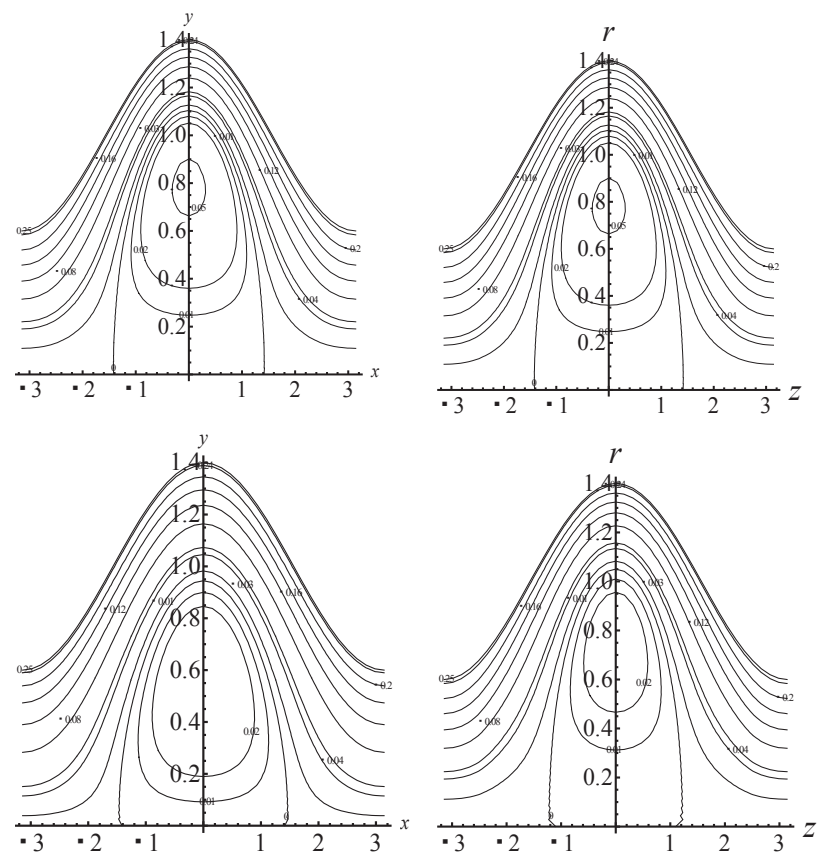

Fig.8. Plots showing the comparison of Streamline in channel and tube when $F=-0.25, \phi=0.4$ and $z=x=\pi$. for $W e=0.5, W e=0.9$ when $\alpha=0.5$
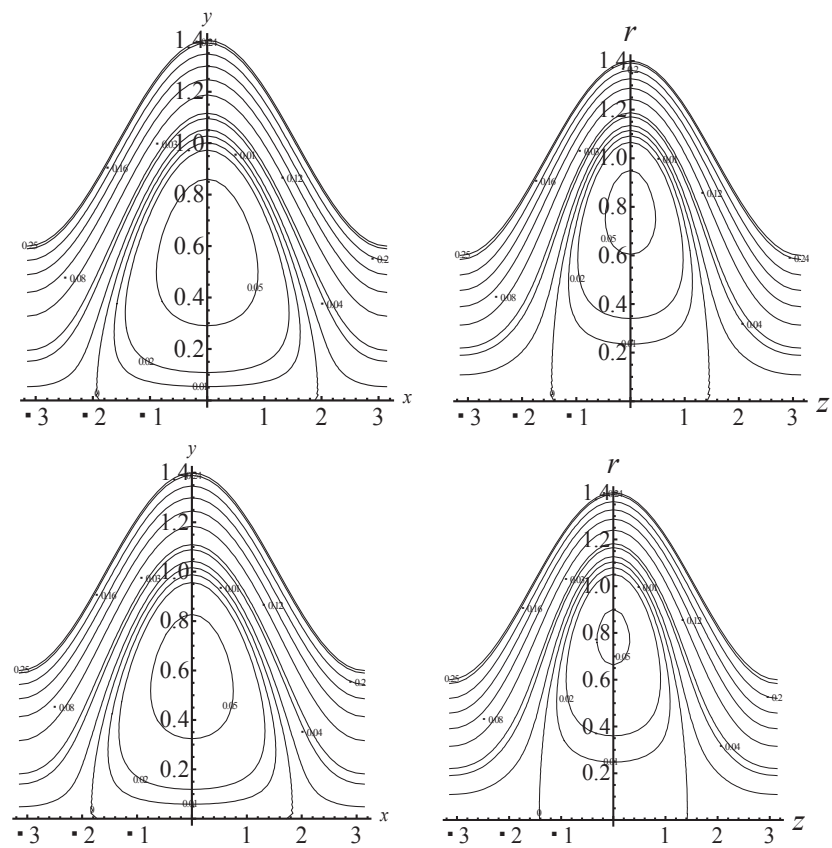

Fig. 9. Plots showing the comparison of Streamline in channel and tube when $F=-0.25, \phi=0.4$ and $z=x=\pi$. for $\alpha=0.3 . \alpha=0.5$. when $W e=0.5$. 


\section{REFERENCES}

1) Latham TW, Thesis MS, MIT, Cambridge (1966).

2) Fung YC, Yih CS, J Appl Mech, 35, 669-675 (1968).

3) Shapiro AH, Jaffrin MY, Weinberg SL, J Fluid Mech, 37, 799825 (1969).

4) Lykoudis PS, Roos R, J Fluid Mech, 43, 661-674 (1970).

5) Pozrikidis C, J Fluid Mech, 180, 515-527 (1987).

6) Takabatak S, Ayukawa K, Mori A, J Fluid Mech, 193, 267-283 (1988).

7) Takabatak S, Ayukawa K, J Fluid Mech, 122, 439-499 (1982).

8) Böhme G, Friedrich R, J Fluid Mech, 128, 109-122 (1983).

9) Raju KK, Devanathan R, Rheol Acta, 11, 170-178 (1972).

10) Raju KK, Devanathan R, Rheol Acta, 13, 944-948 (1974).

11) Srivastava LM, Srivastava VP, J Biomech, 17, 821-829 (1984).

12) Siddiqui AM, Schwarz WH, Rheol Acta, 32, 47-56 (1993).

13) Hayat T, Wang Y, Siddiqui AM, Hutter K, Asghar S, Math Models and Methods in Appl Sci, 12, 1691-1706 (2002).

14) Siddiqui AM, Provost A, Schwarz WH, J Non-Newtonian Fluid Mech, 53, 257-284 (1994).

15) Abd elmaboud, Y, Mekheimer KhS, Appl Math Model, 35, 2695-2710 (2011).

16) Haroun MH, Compu Mat Sci, 39, 324-333 (2007).

17) Rao AR, Mishra M, J Non-Newtonian Fluid Mech, 121, 163 174 (2004).

18) Ali N, Hayat T, Applied Mathematics and Computation,193, 535-552 (2007).

19) Wang Y, Hayat T, Ali N, Oberlack M, Physica A, 387, 347-363 (2008).

20) Vajravelu K, Sreenadh S, Babu VR, Int J Non-Linear Mechanics, 40, 83-90 (2005).
21) Ali N, Abbasi A, Ahmad I, AIP Advances, 5097214 doi: 10.1063/1.4932042 (2015).

22) Srivastava LM, Rheol Acta, 25, 638-641 (1986).

23) Mekheimer KhS, Biorheology, 39, 755-765 (2002).

24) Ali N, Hayat T, Sajid M, Biorheology, 44, 125-138 (2007).

25) Srinivasacharya D, Mishra M, Rao AR, Acta Mech, 161, 165178 (2003).

26) Hayat T, Wang Y, Siddiqui AM, Hutter K, Math Problems Eng, 1, 1-23 (2003).

27) Hayat T,Wang Y, Hutter K, Asghar S, Siddiqui AM, Math. Problems. Eng,4, 347-376 (2004).

28) Wang Y, Hayat T, Huttler K, Theor Comput Fluid Dyn, 21, 369-380 (2007).

29) Ali N, Wang Y, Hayat T Oberlack M, Biorheology, 45, 611628 (2008).

30) Ali N, Wang Y, Hayat T, Oberlack M, Canadian J Phys, 87, 1047-1058 (2009).

31) Ali N, Asghar Z, Z Naturforsch, 69a, 462-472 (2014).

32) Tripathi D, Bég O Anwar Com Meth Bio \& Bio Eng, 10, 10255842:2014:940332 (2014).

33) Giesekus H, J Non-Newtonian Fluid Mech, 11, 69-109 (1982).

34) Giesekus H, JNon-Newtonian Fluid Mech, 12, 367-374 (1983).

35) Bird RB, Armstrong RC, Hassager O, 1, Wiley, New York, (1977).

36) Schleiniger G, Weinacht RJ, J Non-Newtonian Fluid Mech, 40(1), 79-102 (1991).

37) Ali N, Javed T, Z Naturforsch, 68a, 515-523 (2013).

38) Kalantari A, Riasiland A, Sadeghy K, Nihon Reoroji Gakkaishi, 42, 9-17 (2014).

39) Pourjafar M, Sadeghy K, J Non-Newtonian Fluid Mech, 177178, 54-63 (2012). 